\title{
Bias-Dependent Annealing of Radiation Damage in Neutron-Irradiated Silicon $p^{+}-n-n^{+}$Diodes
}

\author{
V. Cindro, G. Kramberger, M. Mikuž, D. Žontar \\ Jožef Stefan Institute and Departement of Physics, University of Ljubljana, \\ SI-1000 Ljubljana, Slovenia
}

\begin{abstract}
Silicon pad diodes fabricated on $n$ type substrates were irradiated with fast neutrons from the TRIGA reactor facility to fluences around $5 \times 10^{13} \mathrm{n} / \mathrm{cm}^{2}$. Annealing of radiation-induced defects was monitored via the full depletion voltage (FDV) obtained from $C-V$ measurements, both during and after irradiation. As much as 50 $\%$ difference in FDV was observed to appear between biased and unbiased diodes in a few hours after irradiation, with the biased diodes having the higher value. The FDV difference persists through later stages of annealing and reverse annealing. Upon switching off the bias voltage, the difference in FDV diminishes within days at room temperature. Observation of different effective dopant concentrations within the same detector in regions with and without electric field provides further evidence for bias-dependent annealing. No bias effect is seen on the reverse-current annealing.
\end{abstract}

The change of effective impurity concentration in silicon, caused by irradiation with fast heavy particles (protons, pions, neutrons), results in a change of the full depletion voltage. Another effect of irradiation is an increase of the leakage current related to the introduction of defects with energy levels near mid-gap. The damage is found to scale with the non-ionizing energy loss (NIEL) of particles. Silicon will be used as the sensing medium for charged particles in trackers and calorimeters at experiments being constructed for the LHC. The increase of full depletion voltage together with the increase of leakage current could result in an electric breakdown, thermal runaway and unacceptable increase in parallel noise, setting a limit on usage of silicon detectors for these applications.

A lot of measurements have been performed in order to predict the level of radiation damage in silicon at LHC experiments. An increase of effective acceptor concentration has been observed during irradiations. This increase, 
followed by annealing and reverse annealing has been extensively studied for pions, protons and neutrons [1-3]. Typically, the full depletion voltage has been obtained from $C-V$ measurements. Most of the samples have been irradiated and their annealing studied without bias. In the LHC experiments, however, detectors have to be under bias during data taking. Therefore, we have set up a study of the effect of bias voltage on changes of full depletion voltage and leakage current induced by neutron irradiation.

The TRIGA nuclear reactor at the Jožef Stefan Institute was used as the neutron source. The damage factor relative to $1 \mathrm{MeV}$ neutrons, calculated from NIEL given by $[4,5]$, was found to be $0.88 \pm 0.05$. Absolute fluence was determined for each irradiation by gold activation with a precision of $\approx 10 \%$. A slightly lower damage factor value of $0.76 \pm 0.04$ was deduced from a direct experimental comparison of leakage currents caused by different particles [6]. The diodes under study were processed by MICRON on high resistivity $300 \pm 5 \mu \mathrm{m}$ thick n-type float-zone silicon wafers. Their properties as well as irradiation parameters are summarized in Table 1. The guard structure on these diodes consisted of two or three guard rings. The measurement of full depletion voltage was limited by breakdown to approximately 500 volts.

\begin{tabular}{||c|c|c|c|c|c|c||}
\hline \hline $\begin{array}{c}\text { Sample } \\
\text { label }\end{array}$ & $\begin{array}{c}\left|N_{\text {eff }}^{0}\right| \\
{\left[10^{12} \mathrm{~cm}^{-3}\right]}\end{array}$ & $\begin{array}{c}\text { Fluence } \\
{\left[10^{13} \mathrm{n} / \mathrm{cm}^{2}\right]}\end{array}$ & $\begin{array}{c}\text { Irrad. time } \\
{[\mathrm{h}]}\end{array}$ & $\begin{array}{c}V_{\text {bias }} \\
{[\mathrm{V}]}\end{array}$ & $\begin{array}{c}\text { T(irr } / \text { ann }) \\
{\left[{ }^{O} \mathrm{C}\right]}\end{array}$ & $\begin{array}{c}\left|N_{\text {eff }}^{\min }\right| \\
{\left[10^{12} \mathrm{~cm}^{-3}\right]}\end{array}$ \\
\hline D1 & 0.24 & 4.7 & 0.65 & 300 & 15 & $/$ \\
D2 & 0.34 & 5.0 & 6.5 & 200 & $5 / 60$ & 1.70 \\
D3 & 0.23 & 4.7 & 6.5 & 200 & $15 / 60$ & 1.76 \\
D4 & 0.29 & 4.9 & 6.5 & 200 & $0 / 60$ & 1.66 \\
D5 & 0.24 & 4.7 & 6.5 & no & $18 / 60$ & 0.99 \\
D6 & 0.27 & 4.7 & 6.5 & no & $18 / 60$ & 0.96 \\
D7 & 0.24 & 4.7 & 6.5 & no & 15 & 0.90 \\
D8 & 0.49 & 5.0 & 6.5 & $*$ & $15 / 28 / 20$ & $/$ \\
D9 & 0.46 & 5.0 & 6.5 & $* *$ & $15 / 28 / 20$ & 0.86 \\
\hline \hline
\end{tabular}

Table 1

Summary of detector propertiess and radiation conditions. $\left|N_{e f f}^{0}\right|$ denotes the initial effective dopant concentration and $\left|N_{e f f}^{\min }\right|$ at the minimum after beneficial annealing. T(irr/ann $)$ is the temperature during irradiation/annealing. All the detector thicknesses were $300 \pm 5 \mu \mathrm{m}$, so the $\left|N_{\text {eff }}\right|$ is proportional to FDV to better than $3 \%$. As all the detectors are inverted to p-type, the change in $\left|N_{e f f}\right|$ is obtained through $\Delta\left|N_{e f f}\right|=\left|N_{e f f}^{0}\right|+\left|N_{e f f}^{\min }\right|$. Samples D1 and D8 were not annealed to the minimum.

* - no bias during, biased to $200 \mathrm{~V}$ after irradiation

** - biased to $200 \mathrm{~V}$ during, no bias after irradiation 


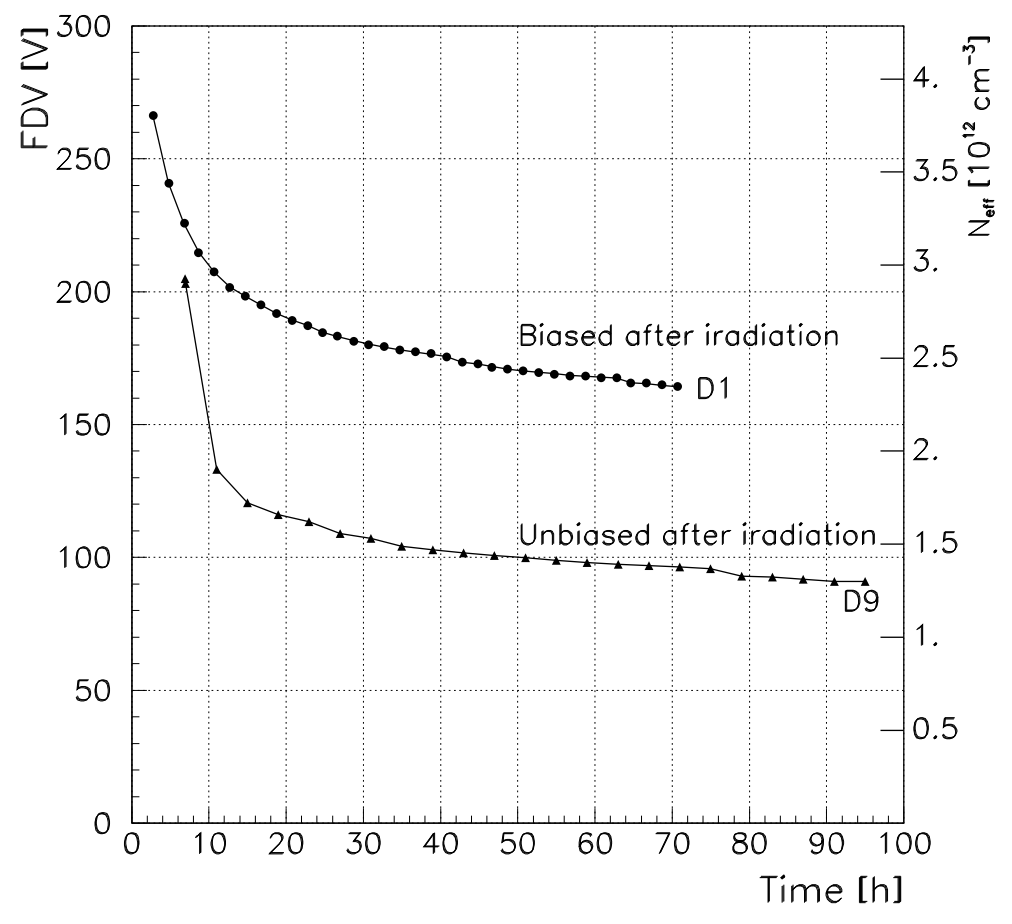

Fig. 1. $\left|N_{\text {eff }}\right|$ and FDV of biased and unbiased diode as function of time after beginning of irradiation.

A comparison of short term (several hours) annealing behaviour after irradiation to $5 \times 10^{13} \mathrm{n} / \mathrm{cm}^{2}$ of two differently biased MICRON ${ }^{1}$ samples is shown in Fig. 1. D1 was biased to $300 \mathrm{~V}$ during and after irradiation, while D9 was kept at $200 \mathrm{~V}$ during irradiation and unbiased after irradiation. Both diodes were kept at $15^{\circ} \mathrm{C}$, a frequency of $10 \mathrm{kHz}$ was used in $C-V$ measurements. After the initial stage of annealing, the diode unbiased after irradiation exhibits a lower $\left|N_{\text {eff }}\right|$ than the biased one, by far exceeding the difference in $\left|N_{e f f}\right|$ before irradiation. The difference in $\left|N_{\text {eff }}\right|$ at the end of irradiation can be explained by the annealing during irradiation. The irradiation time was 39 minutes for D1 and 6.5 hours for D9, the latter being in the same range as short-term annnealing time constants. This irradiation-time difference plays no role for defects with annealing times longer than a few days. Further, in Ref. [7], we ruled out a possible flux dependence of FDV for diodes irradiated under the same bias conditions. Therefore, the only way to explain the difference in the $\left|N_{\text {eff }}\right|$ between the two samples is by bias-dependent short-tem (few hours) annealing of some radiation-induced defects.

The bias effect after longer term annealing was also studied. Annealing was accelerated by heating the diodes to $60^{\circ} \mathrm{C}$. Figure 2 shows the time evolution of $\left|N_{\text {eff }}\right|$ inferred from FDV measurements at $5^{\circ} \mathrm{C}$ and $10 \mathrm{kHz}$. The samples

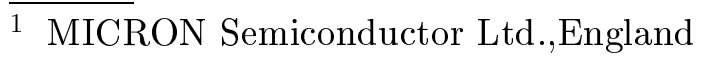




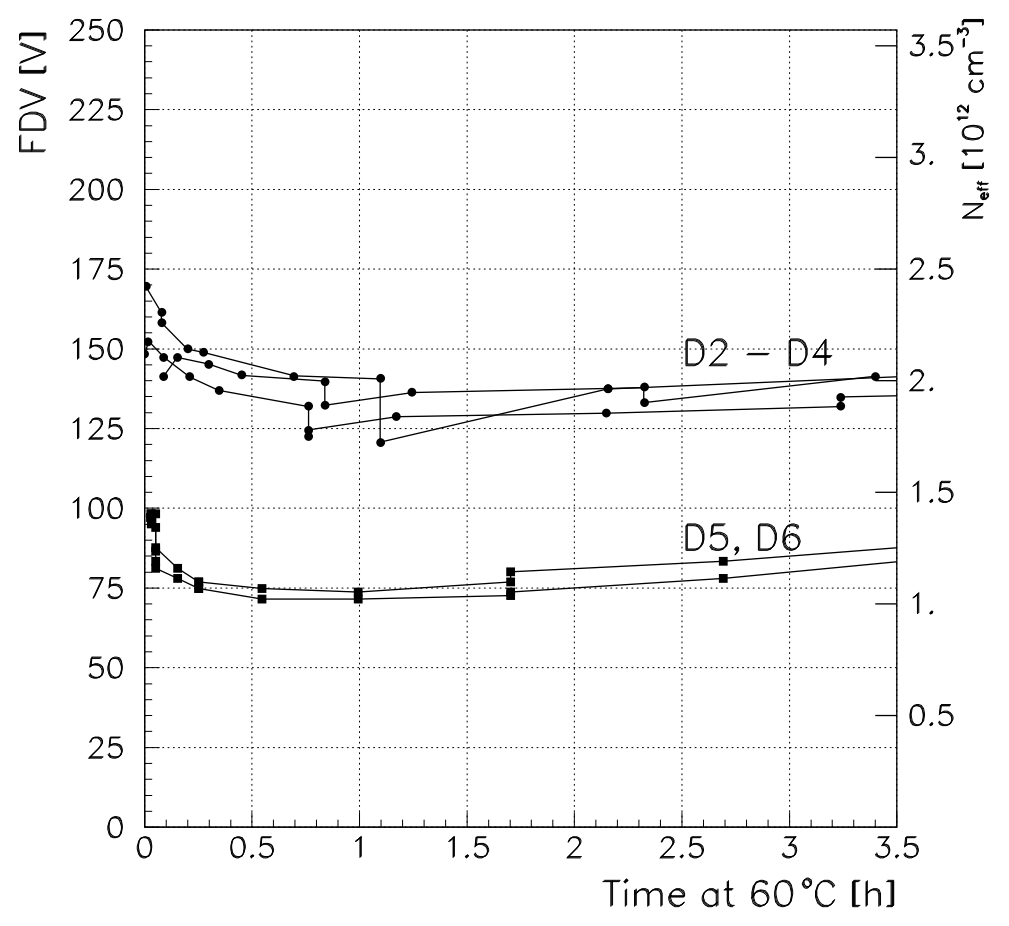

Fig. 2. $\left|N_{\text {eff }}\right|$ and FDV of biased (D2-D4) and unbiased (D5,D6) diodes during accelerated annealing at $60^{\circ} \mathrm{C}$. Measurements were done at $5^{\circ} \mathrm{C}$.

shown were irradiated to $5 \times 10^{13} \mathrm{n} / \mathrm{cm}^{2}$, irradiation time of all diodes was 6.5 hours. Diodes D2-D4 were biased all the time, while diodes D5 and D6 were kept unbiased during and after irradiations.

We observe that biased diodes have a FDV of about $130 \mathrm{~V}$, compared to $75 \mathrm{~V}$ for the diodes that were irradiated and annealed without bias. The respective difference in $\left|N_{\text {eff }}\right|$ is about $0.8 \times 10^{12} \mathrm{~cm}^{-3}$, with differences in initial $\left|N_{\text {eff }}^{0}\right|$ below $0.1 \times 10^{12} \mathrm{~cm}^{-3}$. This shows that the bias effect persists through longterm annealing when the minimum in FDV is reached. Combining this result with the result from Fig. 1 we conclude that most of the difference in $\left|N_{\text {eff }}\right|$ between biased and unbiased samples is generated during short-term annealing on a timescale of several hours.

For the impact of this effect on the operation of detectors at the LHC, it is vital to check, whether this additional damage anneals out when the bias is switched off. In Fig. 3, the D8 diode was irradiated without bias and annealed under bias (200V). After 13 days of annealing, when most of beneficial annealing was completed, the bias on this diode was switched off. For a comparison, unbiased annealing of D9 is shown in the same figure.

Both diodes were mounted on the same substrate, so they were irradiated and annealed under exactly the same conditions except for the bias. FDV of D8 


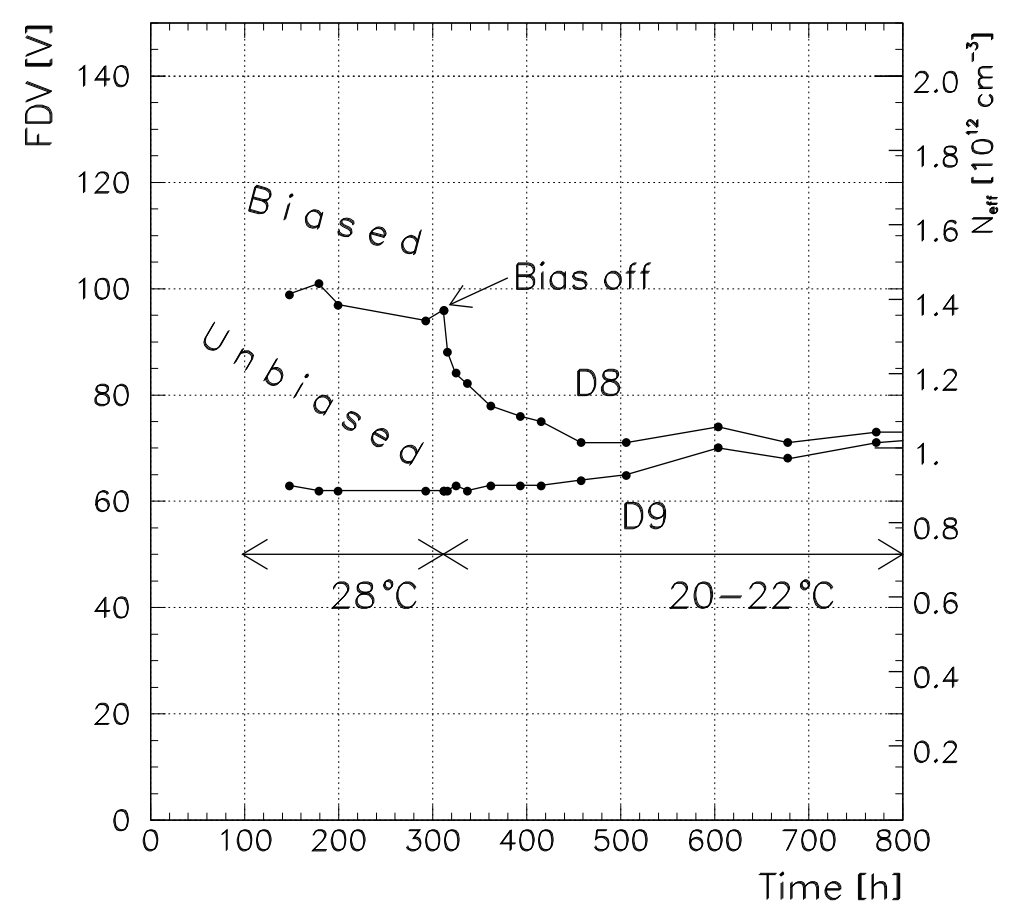

Fig. 3. FDV after switching off the bias (D8). For a comparison the unbiased diode (D9), irradiated and annealed in parallel, is shown.

during annealing under bias is somewhere between the FDV's of biased and unbiased diodes shown in Fig. 2, since a part of fast annealing, that is inhibited by the bias, had already taken place during the $6.5 \mathrm{~h}$ irradiation without bias. We observe that the additional damage anneals after the bias is switched off and FDV of D8 comes close to that of D9.

We found additional evidence for bias dependent damage in $C-V$ measurements of reverse-annealed diodes. The $1 / C^{2}-V$ characteristics of such a diode, irradiated to $10^{14} \mathrm{n} / \mathrm{cm}^{2}$, is shown in Fig. 4. The data with full circles represent the measurement after 21 days of annealing at $60^{\circ} \mathrm{C}$ and with a constant bias of $200 \mathrm{~V}$, thus most of the reverse annealing already took place. $C-V$ measurements were performed at $5^{\circ}$. A kink in the slope is present at around $200 \mathrm{~V}$, the slope changing by a factor of two. This indicates double effective dopant concentration in the depleted part relative to the undepleted part of the diode. After switching off the bias and keeping it at room temperature for 3 days, the line with open circles is obtained. The slope of the previously depleted region is diminished. This means that defects whose annealing is inhibited by the electric field, remain in silicon also during long term reverse annealing. They start annealing out, however, upon switching off the bias.

A bistable defect, generated by heat treatment of irradiated silicon, with a bias-inhibited annealing behaviour was observed and studied in [8]. Its decay 


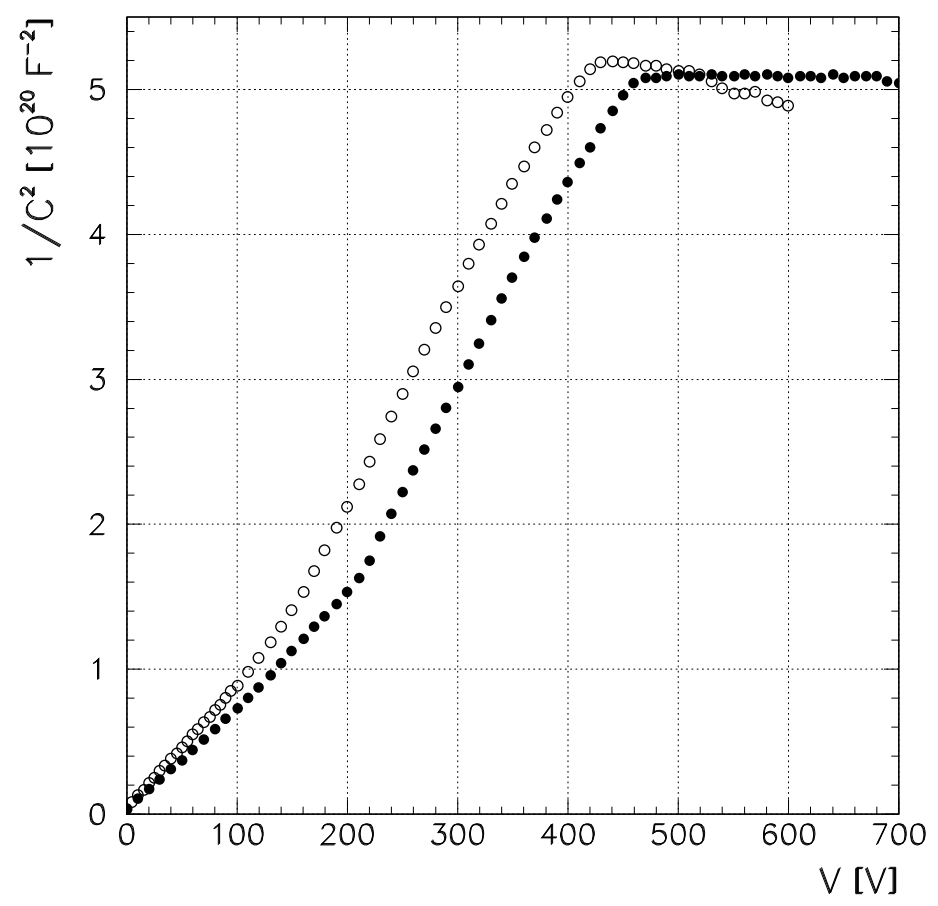

Fig. 4. 1/ $C^{2}-V$ characteristic of a permanently biased diode after reverse annealing (full circles). After several days bias had been switched off, we measured the characteristic represented by open circles.

time at $20^{\circ} \mathrm{C}$ was 8.5 hours, the activation energy being $1.04 \mathrm{eV}$. It could be re-activated by forward current, re-heating or light illumination. Its presence was, however, observed only upon sufficient (at $80^{\circ} \mathrm{C}$ for more than 10 hours) heating of reverse-annealed samples, while our defect is clearly present already right after irradiation at low temperature (Fig. 1). Moreover, our bias-off annealing at $20^{\circ} \mathrm{C}$ in Fig. 3 seems to exhibit also a longer-term component, so further detailed studies are needed to make a definite comparison of the two observations.

No bias effect was observed in the leakage current annealing. This is demonstrated in Fig. 5 where the annealing of the leakage current constant is plotted. There is no systematic difference between the behaviour of unbiased and biased samples. This fact can be explained by the energy level of the bias-dependent defect being far from the mid-gap, thus bearing no influence on the generation current.

To conclude: we have experimentally demonstrated different annealing behaviour of effective dopant concentrations of biased and unbiased diodes after neutron irradiation. This could be related to the fact that irradiation induced deep deffects may be neutral in an undepleted detector and become charged when the detector is depleted. Switching off the bias at room temperature 


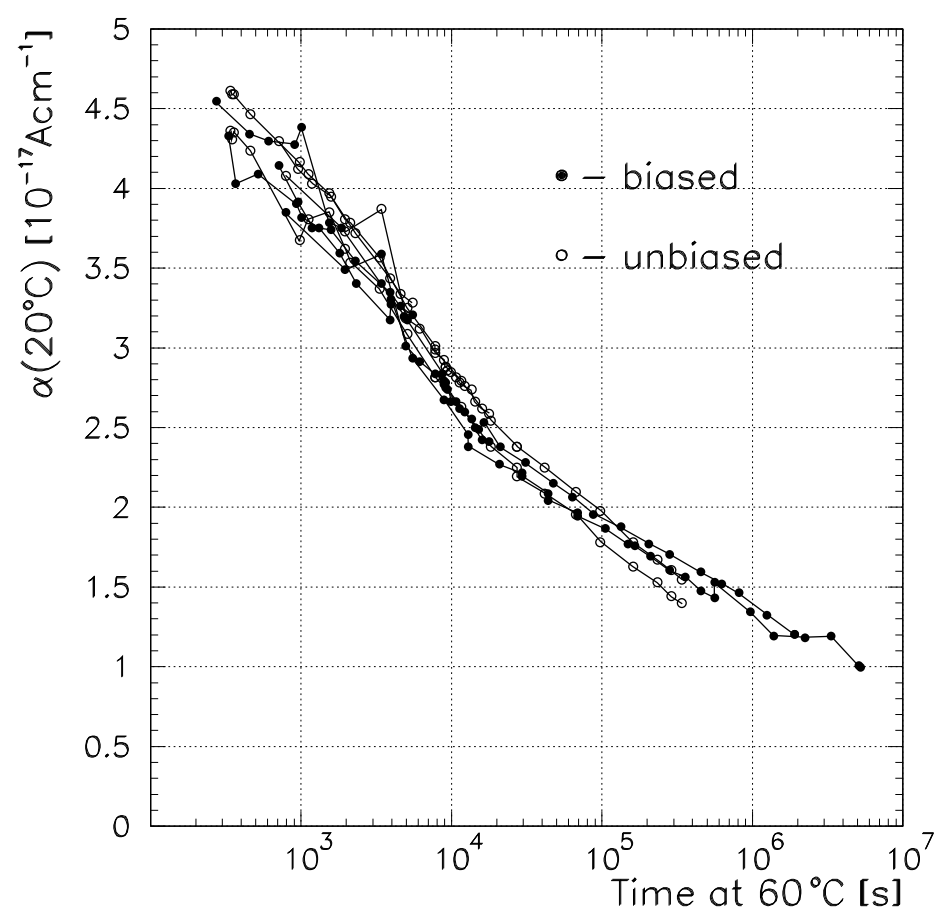

Fig. 5. Annealing of leakage current for biased (closed circles) and unbiased (open circles) diodes.

tends to recover the detector after a few days. We might think that LHC detectors could be anyway unbiased for a sizable portion of the time to recover this additional damage. However, from Fig. 3 we note that the recovery time at $20^{\circ} \mathrm{C}$ coincides with the onset of reverse annealing so that an optimal operating scenario will depend crucially on the activation energy of the biasdependent effect(s). In addition, we should note that the bias on our samples was not sufficient to fully deplete the detectors immediately after irradiation. Thus a part of the bias-inhibited fast annealing had already taken place in the undepleted part of the silicon. Therefore, further detailed studies are needed to arrive to more reliable predictions of the influence of bias on the full depletion voltage.

\section{References}

[1] RD2 collaboration: S.J. Bates et al., Nucl. Instr. and Meth. A379(1996) 116.

[2] A. Chilingarov et.al, Nucl. Instr. and Meth. A360(1995) 432.

[3] H. Feick et.al, Nucl. Instr. and Meth. A377(1996) 217.

[4] A.M. Ougouag et al., IEEE Transactions on Nuclear Science, NS-37 (1990) 22192228. 
[5] M.S.Lazo et al., Proc. Fast Burt. React. Workshop, Sandia National Laboratories 1987, SAND-87-0098 Vol.1 85-103.

[6] M. Moll et al., Leakage current of hadron irradiated silicon detectors - matreial dependence (Presented at $2^{\text {nd }}$ International Conference on Radiation Effects on Semiconductor Materials, Detectors and Devices, Florence 1998, submitted to NIM)

[7] D.Žontar, V. Cindro, G. Kramberger and M. Mikuž, (Presented at $2^{\text {nd }}$ International Conference on Radiation Effects on Semiconductor Materials, Detectors and Devices, Florence 1998, submitted to NIM)

[8] M. Moll et al., Nucl. Phys. B (Proc. Suppl.) 44(1995)468. 